\title{
The Effect of a Vertical Magnetic Field on the Periods of Trapped $g$-modes in White Dwarfs
}

\author{
Bradley W. Carroll \\ Dept. of Physics, Weber State University, Ogden, UT 84408-2508
}

\begin{abstract}
A white dwarf model with cylindrical symmetry is used to investigate the effect of a uniform vertical magnetic field $\left(\vec{B}=B_{\circ} \hat{z}\right)$ on the periods of $g$-modes trapped in the compositionally stratified surface layers. For those modes for which trapping is most effective, the periods wander by approximately $10 \%$ about their zero-field values as $B_{0}$ increases. As the field strength increases further, the period may abruptly increase by some $25 \%$ to a new, more stable value; see Figure 1. The periods of less efficiently trapped modes do not show this sensitivity for field strengths up to $1 \mathrm{MG}$, the upper limit examined.
\end{abstract}

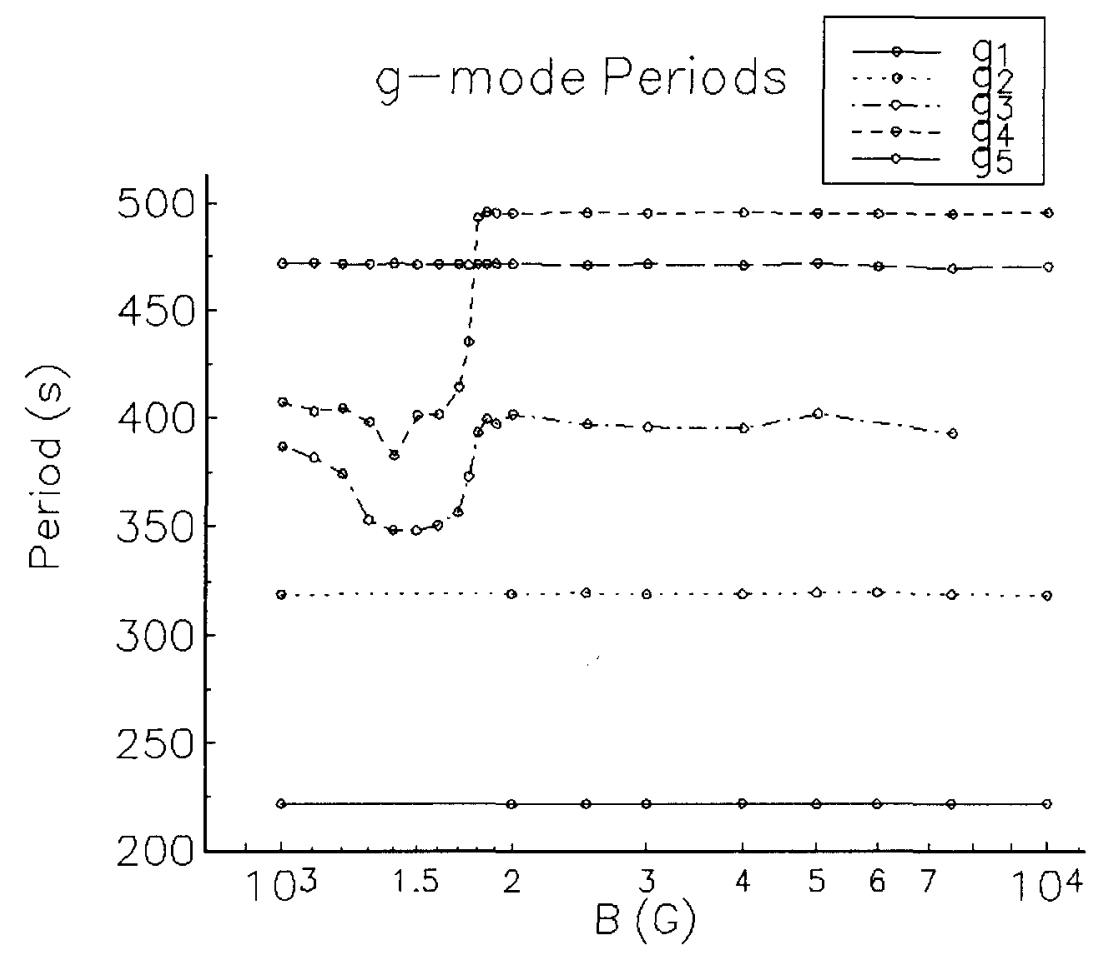

Figure 1 Pulsation periods of $g_{1}-g_{5}$ modes with $\ell=2$ for a compositionally stratified $0.6 \mathrm{M}_{\odot}$ white dwarf $\operatorname{model}\left(T_{e}=9960 \mathrm{~K}, M_{\mathrm{He}} / M_{*}=10^{-5}, M_{\mathrm{H}} / M_{*}=\right.$ $\left.10^{-10}\right)$ 